\title{
Simulation-based Data Analysis to Support the Planning of Flexible Manufacturing Systems
}

\author{
Marc-André Filz* , Christoph Herrmann, Sebastian Thiede
}

Institute of Machine Tools and Production Technology, Chair of Sustainable Manufacturing and Life Cycle Engineering, Technische Universität Braunschweig, Langer Kamp 19 b, 38106 Braunschweig, Germany *m.filz@tu-braunschweig.de

SNE 30(4), 2020, 131-137, DOI: 10.11128/sne.30.tn.10531 Received: August 3, 2020 (Selected ASIM SPL 2019 Postconf. Publ.); Revised: Oct. 19, 2020; Accepted: Oct.20, 2020

SNE - Simulation Notes Europe, ARGESIM Publisher Vienna ISSN Print 2305-9974, Online 2306-0271, www.sne-journal.org

Abstract. A matrix-structured manufacturing system represents a flexible manufacturing system that combines volume- and variant-flexible production and strives for efficiency. The system is a modular, cycle-independent manufacturing concept in which all workstations are linked by a flexible transport system. Due to the function of the manufacturing system, the requirements at the individual workstations are not known in advance. Therefore, the planning of the material supply according to requirements is a relevant target value in the planning and design of such a flexible manufacturing system. In order to support the planning, the characteristics of material supply can be investigated with the help of an agentbased simulation. However, the simulation results must be examined in more detail in order to be used for planning flexible manufacturing systems. Therefore, data analysis can be used to derive necessary knowledge from the simulation data. In this context, the aim of this paper is to support the planning of flexible manufacturing systems by developing and validating a simulation-based data analysis framework.

\section{Introduction}

The line-oriented production is the main production principle in numerous industrial companies. Considering these companies in detail, a variety of challenges can be identified. Among these are a strongly fluctuating demand, decreasing product life cycles and an increasing amount of variants [1].
These influences increase the flexibility and efficiency requirements for the production system. The concept of a matrix manufacturing system (MMS) helps to meet the aforementioned challenges with a high degree of flexibility and scalability [2]. Figure 1 provides the graphical comparison of both manufacturing systems.

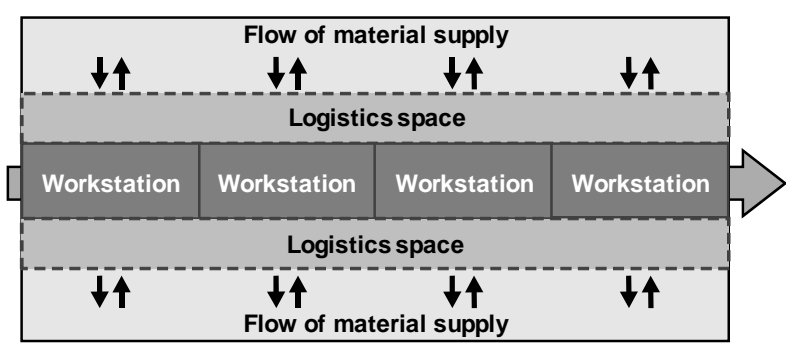

a) $\downarrow \uparrow$ Flow of material supply
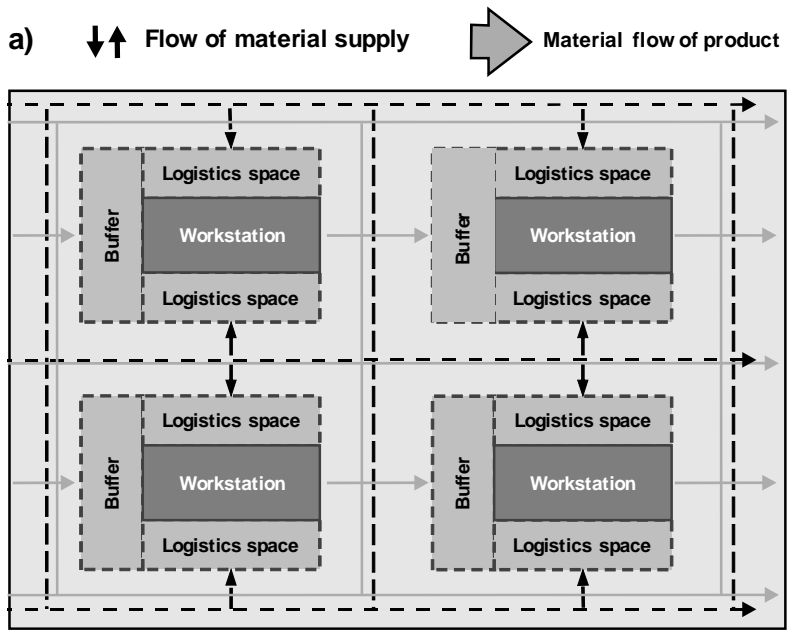

b) $\longrightarrow$ Material flow of product $\quad \rightarrow$ Flow of material supply

Figure 1: Comparison of (a) line configuration and

(b) matrix manufacturing system [3].

The matrix-structured manufacturing system is composed of modular and decoupled workstations (WS), which are internally connected by a flexible transportation system (Figure 1). 
The material flows are coordinated by an intelligent production control system within the manufacturing system.

An elementary component of every production system is the internal material supply, which provides the required components. This process has significant influence on the efficiency of the whole production system. The network-related structure of the MMS consists of an uncoupled modular and redundant WS design. This design leads to multiple demand locations for the same material or modules. Therefore, the destinations and individual routing of the materials are not known beforehand. The assignment of products to the WS is performed by the production control system at short notice and is particularly dependent on the specific circumstances within the manufacturing system. This limits the planning of material supply and reinforces the need for responsiveness and flexibility [3].

The design of this manufacturing approach causes numerous dynamic and stochastic effects. These increasing uncertainties of the system have a direct effect on the material supply strategy. To overcome these challenges already during the planning phase, this paper develops a methodology that uses simulation results as a basis for data analytics to identify and control relevant system parameters in an early planning stage of a flexible manufacturing system using an MMS as an example.

\section{Planning of Matrix Manufacturing Systems}

In order to specify the MMS planning challenges in more detail, the requirements on material supply strategies are explained in Section 1.1. In addition, Section 1.2 discusses different approaches to simulating MMS and derives the need for further research with a specific focus on the material supply strategy within the MMS.

\subsection{Requirements on Material Supply Strategies}

The material supply is a central component of the MMS, because - in contrast to line configuration - no materials are stocked at the individual work stations. Therefore, in order to determine the material supply requirements within the MMS, the influencing factors on the one hand and the design on the other hand must be determined. Due to the fact that the material supply belongs to the logistics system, it is part of the higher-level production system.
Thus, the characteristics of the production system define the framework conditions for the design of the material supply. Furthermore, the characteristics of the material spectrum to be provided have a major influence on the design of the material supply. These include logistical (e.g., frequency of use), physical (e.g., volume) and handling (e.g., bulk material) properties of the material spectrum to be provided [4]. In order to gain a better understanding of the differences between the line configuration and the MMS, Table 1 shows the central characteristics of the respective manufacturing system configurations.

\begin{tabular}{lll}
\hline Requirements & $\begin{array}{l}\text { Line } \\
\text { configuration }\end{array}$ & $\begin{array}{l}\text { MMS } \\
\text { configuration }\end{array}$ \\
\hline $\begin{array}{l}\text { Delivery } \\
\text { locations }\end{array}$ & single & multiple \\
$\begin{array}{l}\text { Material } \\
\text { bundling }\end{array}$ & $\begin{array}{l}\text { dependent on } \\
\text { assembly order }\end{array}$ & hardly possible \\
$\begin{array}{l}\text { Short-term } \\
\text { capacity planning }\end{array}$ & fixed & $\begin{array}{l}\text { Variable / } \\
\text { dynamic }\end{array}$ \\
\hline
\end{tabular}

Table 1: Comparison of selected design parameters for line configuration and MMS [3].

The design of the MMS network structure of unlinked modular workstations and the multi-redundant structure lead to several possible delivery locations for the same material or modules. This requires a simple WS layout, which enables an easy design of the supply of the respective WS. Furthermore, the source-sink relationships are dependent on the respective system status and the selected production control logic in an MMS, because they need to react variably and dynamically. This results in different supply locations for individual workpieces (WP) within a system. Due to the missing planning base as a result of the dynamic system behaviour, a bundling of required materials for supply is hardly manageable [3].

Once the material is fed into the production system, the WS on which the product is processed are not known as they are determined by the production control system on short notice and depending on the individual situation. This leads to a renunciation of short-term material supply planning [3].

In addition, due to the lot size of one, the number of transport operations within the MMS increases. Due to the architecture of the MMS as a network structure, there is an overlap between the product transport and the material supply. 
Assuming a high number of transport processes, the risk of blockages on the transport routes increases significantly, resulting in increased uncertainties in the overall manufacturing system. In this context, deadlocks can occur especially in close-meshed layouts. For example, decentrally controlled automated guided vehicles (AGV) trigger a circular closing and completely block each other. This must be prevented; otherwise, the entire system will be blocked due to missing material supply and product transport [5].

\subsection{Simulation of Material Supply Strategies within MMS}

Simulation is applied to support the planning process of production systems. For this purpose, simulation is used especially in industry and research as a method of representing and imitating real systems as a function over time [6]. Simulation is often used to validate, analyse, and optimise flexible systems, such as production logistics systems [7]. In manufacturing, methods of simulation are used for a wide range of tasks. Most common are layout design, planning, analysis, and optimisation of manufacturing systems [8]. Furthermore, simulation offers the chance to analyse cause-effect relationships within the system and to represent the system behaviour in a comprehensible way $[6,8]$.

Greschke et al. introduce a methodology that strives to combine flexibility and profitability for assembly lines. Therefore, identical cycle times of all products are eliminated and the process is kept smooth. By focussing on a systematic assignment of several operations to specifically equipped WS and by controlling the corresponding distribution to ensure the dynamic configurability of the system, the MMS design is implemented [2].

Following this approach, Schönemann et al. more specifically focus on the main principles, elements, and control strategies of the MMS. Therefore, a simulation approach is introduced and discussed to evaluate different MMS control strategies. A use case is applied to validate the simulation approach for the planning of MMS. However, no detailed consideration is given to material supply strategies within the MMS [9].

Buth et al. focus on agent-based simulation approaches to increase the flexibility of manufacturing systems by using industrial grade software tools. The authors, therefore, introduce a generic methodology for implementing agent-based logic on an MMS use case [10].
Focussing more specifically on logistics, Kern et al. introduce five different material supply strategies for the future modular final assembly in automotive manufacturing. Applying this concept in a use case of pre-assembly at a German automobile manufacturer, a space reduction of $15 \%$ could be achieved. However, the performance of the concept has not undergone any validation [11].

Filz et al. built up on the concept of Schönemann et al. [9] and expanded the MMS simulation approach with a focus on material supply strategies. Therefore, different material supply strategies are modelled and analysed with regard to predefined key performance indicators in an agent-based simulation environment [3].

Nevertheless, none of these approaches regarding the agent-based simulation of MMS anticipate decision support under consideration of uncertainties for the planning of material supply strategies within an MMS. Moreover, none of these approaches can help to understand and gain insight from various simulation runs. Consequently, the planning process of highly flexible systems like the MMS cannot be supported by previously validated planning parameters of the material supply system.

\section{Data Analysis Framework for Planning of Flexible Manufacturing Systems}

Previous investigations regarding simulation of flexible manufacturing systems, such as the MMS, show that these systems are highly dynamic and hardly predictable. This impedes the planning process of such systems. In order to support the planning process from an engineering perspective, decision support for relevant planning parameters is necessary. Therefore, a framework is presented in the following that supports the planning process of flexible manufacturing systems by applying a data analysis model on simulation results. The aim of this model is deriving knowledge based on simulation data in order to draw conclusions and to gain insight into interdependencies between different parameters, providing decision support for the planning of such manufacturing systems. In this context, decision support is understood as a target-oriented analysis of the simulation results under consideration of the stochastics and uncertainties of the manufacturing system for the most robust derivation of sensitivities and, thus, design parameters for the manufacturing system. 


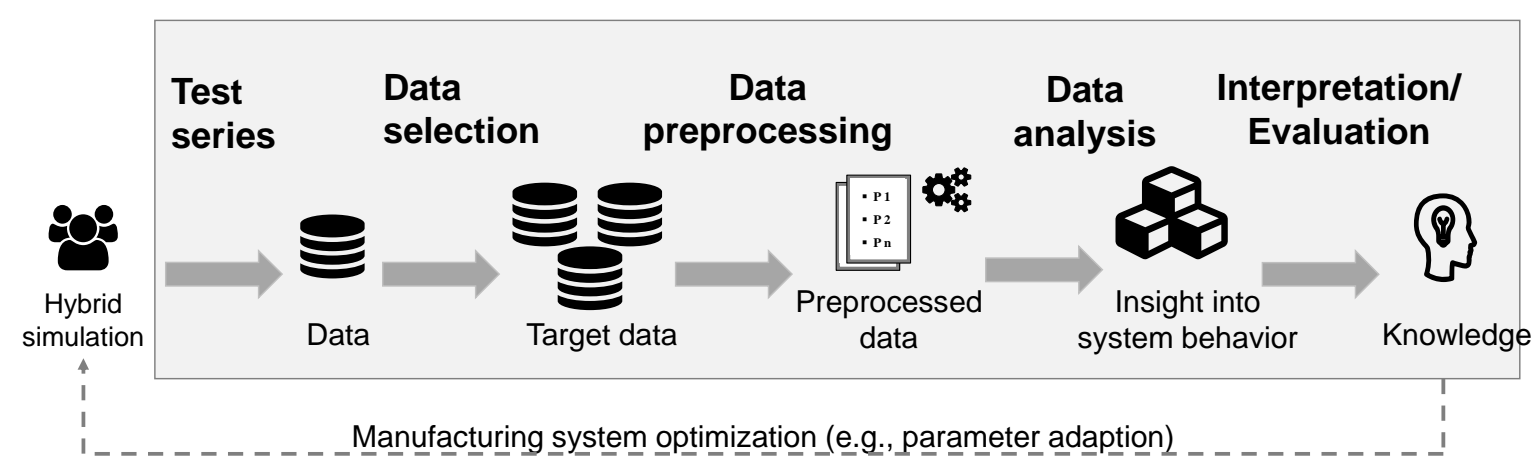

Figure 2: Data analysis framework for planning of flexible manufacturing systems (based on [12]).

The framework is based on the knowledge discovery in databases approach of Fayyad et al. as a standardized and widely-used procedure [12]. Moreover, it is extended to the specific requirements and application of simulation-based data.

The data analysis framework for planning of flexible manufacturing systems within this paper consists of several steps that are shown in Figure 2.

Initially, a simulation model has to be built. Since the focus of this paper is on the data analysis of simulation results, the development and validation of a simulation model is neglected.

Within the first step of the developed framework for simulation-based data analysis, necessary data for further analysis have to be generated. Therefore, various simulation test series have to be carried out for data generation. Since the manufacturing system is subject to stochastic and dynamic behaviour, the data used for analysis need to reflect the most representative system behaviour. Therefore, several target parameters that are relevant for the planning of the specific system have to be detected. In order to gain a deeper understanding of the system behaviour and the influence as well as interactions of the individual parameters, a parameter variation with corresponding simulation runs has to be carried out. Thereby, the behaviour of the individual parameters as well as the entire system can be monitored. In order to create the basis for a subsequent analysis, it is necessary to have a sufficient number of simulation runs that cover all necessary parameter combinations. Furthermore, the simulation results must be saved in a way that allows for subsequent analysis.

After acquiring simulation results, the target data need to be selected. For this purpose, the data are analysed regarding their importance for a larger scheme or system (e.g., utilisation of manufacturing system).
In this context, clustering methods can be used for unsupervised learning or classification for supervised learning approaches. The advantage of a clustering approach lies in the fact that automated and comprehensible thresholds between different parameter ranges can be defined. The choice of approach depends on the respective application [12].

The target data sets identified in this process will be used for further analysis. Moreover, the previously identified data sets are examined for their effect on the target value. Therefore, a correlation analysis of the parameter regarding the target value (e.g., utilisation of manufacturing system) is performed. The parameters with the highest correlation coefficient regarding the target value are considered for further steps.

Furthermore, the collected data need to be preprocessed to ensure data quality. Thus, the data must be converted into a format that allows for further processing. This includes, for example, the xlsx or CSV format. With the help of data structuring, the collected data are transformed into processable data types. In addition, the data need to be formatted to make them suitable for further analysis. Nevertheless, the data are filtered with respect to the analysis aim (e.g., selection of material-supply-related data).

The data analysis is supposed to offer insights into interdependencies between different parameters and their effect on the overall manufacturing system. For this purpose, the sensitivity of the parameters regarding the target value is analysed. Principally, different methods of data analysis can be used for this, such as data mining. On the one hand, this can be used for quantifying and ranking the effect of individual parameters on the target value. On the other hand, the identified parameter ranges can be used to obtain information on how such a system is to be planned in order to obtain optimal utilisation or performance. 
In this context, for example, box plots as statistical methods can be used to analyse the distribution of the parameters with regard to their target value. A boxplot is a diagram to display the distribution graphically. With the help of a boxplot a first impression about the location and the distribution of data in a certain range can be given [13].

Within this developed framework, the data analysis aims at determining the optimal parameter combinations and their ranges. By using data analysis methods, parameter interdependencies can be identified and be provided as input and support for planning purposes. The tool is designed for the usage by different application groups such as planning or quality engineers.

With the help of the data analysis, knowledge as decision support is available, in which exact influences of individual parameters as well as parameter ranges and sensitivities are defined to reach the optimal target value.

For the improvement of the planning process of flexible manufacturing systems, the acquired knowledge can be used to improve the simulation model as a feedback loop as well as to support the overall planning process of efficient and flexible manufacturing systems.

\section{Application of the Decision Support Model on a Matrix Manufacturing System}

Since the MMS is highly flexible and dynamic, it is a hardly predictable system. This sets particularly high demands on the material supply system in terms of flexibility and responsiveness. Therefore, the previously introduced framework for decision support for flexible manufacturing systems is applied to the planning of the material supply strategy within the MMS.

For this purpose, an existing hybrid simulation model for the MMS with a focus on different material supply strategies will be used for simulation-based data analysis. The simulation model is based on existing work by Filz et al. [3].

After implementing the simulation model, multiple simulation runs are performed with a focus on the respective material supply strategy to generate test series. Therefore, a parameter variation is carried out that considers central planning parameters of the logistics system. During this process, the overall utilisation of the production system is set as a target value that describes the average working time of all individual ressources (e.g., machines) within the entire manufacturing system.
Each simulation run was carried out with 500 products. In total, about 3,000 simulation runs were carried out that cover the combinations of the selected parameters. Moreover, the simulation results were saved in an Excel spreadsheet for further processing. Table 2 gives an overview of the selected parameters for the parameter variation with their respective minimum and maximum values as well as the iteration steps.

\begin{tabular}{llll}
\hline Parameter & $\begin{array}{l}\text { minimal } \\
\text { value }\end{array}$ & $\begin{array}{l}\text { max.imal } \\
\text { value }\end{array}$ & $\begin{array}{l}\text { Step } \\
\text { size }\end{array}$ \\
\hline $\begin{array}{l}\text { Amount of products } \\
\text { in system }\end{array}$ & 6 & 13 & 1 \\
Amount of AGV & 2 & 18 & 1 \\
$\begin{array}{l}\text { Velocity of AGV } \\
\text { [m/s.] }\end{array}$ & 0.5 & 1.5 & 0.1 \\
$\begin{array}{l}\text { Loading time AGV } \\
\text { Unloading time AGV }\end{array}$ & 25 & 35 & 2 \\
\hline
\end{tabular}

Table 2: Selected parameter variation for test runs.

Within the framework, the target data are identified in a first step. In this use case, a k-means clustering method with six clusters is applied in order to identify the target data sets with a high utilisation of the manufacturing and logistic system. In addition, clustering is used to provide a transparent separation between the data. Therefore, a threshold at 0.19 for the overall utilisation of the manufacturing system is set. Consequently, only data sets that lead to a utilization of the manufacturing system over 0.19 will be used for further analysis (Figure 3a).

Furthermore, a correlation analysis of the filtered data is performed to identify the parameters with the highest impact on the target value of the overall manufacturing system utilisation.

Figure $3 \mathrm{~b}$ displays the results of the correlation analysis in a ranked order. Based on this, the parameter "Amount of AGV" has the highest correlation coefficient with 0.62 , followed by "Velocity of AGV" with 0.22 and "Amount of products in system" with 0.11 .

For the purpose of data preprocessing, the selected data are checked for formal criteria such as data type in a first step. Within the preprocessing, the removal of noise or handling missing values within the target data sets is extremely important in order to be able to carry out the necessary calculations efficiently and in a target-oriented way. 


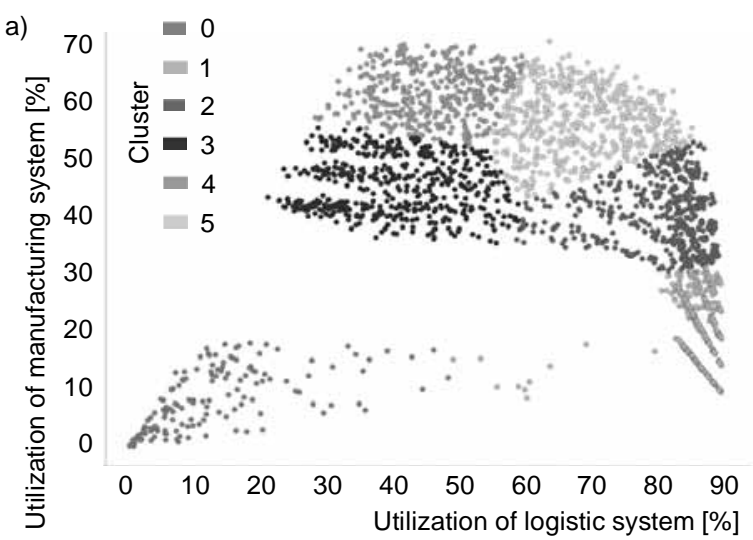

b)

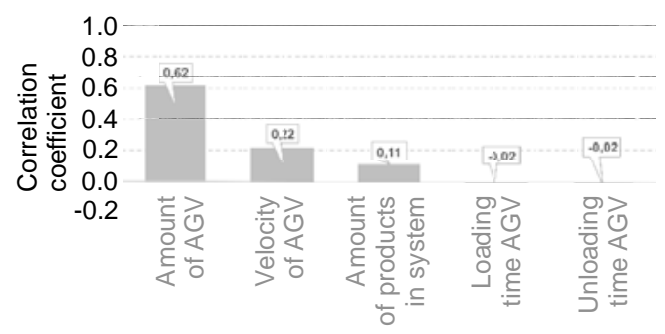

Figure 3: Identification of target data:

(a) Data clustering for defining threshold;

(b) Ranking of parameters based on correlation coefficient.

Within the data analysis step, a sensitivity analysis is performed. The previously selected parameters are analysed with the help of a boxplot to further identify at what range the parameters need to be set to ensure a high utilisation of the manufacturing system. Figure 4 displays the boxplots of the identified parameters in relation to the target value as a basis for sensitivity analysis.

Figure 4a graphically displays the behaviour of "Velocity of AGV" with the regard to the "Overall utilisation of the manufacturing system". The results show that the parameter values fluctuate between 0.2 and 0.7 of the utilisation of the manufacturing system.
However, the median changes only slightly during the increasing velocity of the AGV. Therefore, the parameter is seen as not very sensitive. With regard to the results, the highest utilisation of the manufacturing system can be achieved with a "Velocity of AGV" between 1.1 and 1.3.

With the help of Figure 4b, the sensitivity of "Amount of products" can be analysed. The simulation study assumed that a minimum of 6 and a maximum of 13 products are simultaneously in the system. The results show that with a higher amount of products the utilisation of the manufacturing systems increases. However, it should be pointed out that further research has shown that there is saturation for about 11 products; otherwise, there will be longer waiting times at the WS that lead to a decreasing utilisation of the system, and a higher number of transportation systems (e.g., AGV) is required. This increases the risk of deadlocks.

Figure $4 \mathrm{c}$ displays an increasing utilisation of the manufacturing system with increasing "Amount of AGV". A saturation of the utilisation can be determined between 9 and $11 \mathrm{AGV}$. This results in the fact that an additional provision of $\mathrm{AGV}$ does not lead to an increase of the utilisation of the manufacturing system and can, therefore, not be recommended.

After the individual parameters have been analysed with regard to their behaviour on the target value, the next step is to determine the optimal parameter combination. Within this use case, the assumption is made that the utilisation rate in such a flexible manufacturing system should be over 60 percent. To determine the optimal parameter combination, a decision tree is used to optimise the overall utilisation. With regard to the planning of the material supply within a flexible manufacturing system, the results show that the combination of 9 to $11 \mathrm{AGV}$ with a velocity between 0.75 and $0.95 \mathrm{~m} / \mathrm{s}$ seems to be the most robust combination. Ideally, there will be 9 to 11 products within the system at the same time. a)

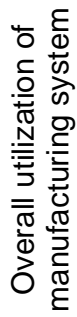

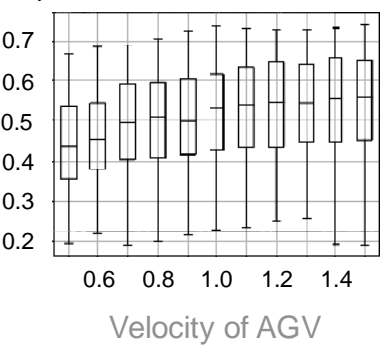

b)

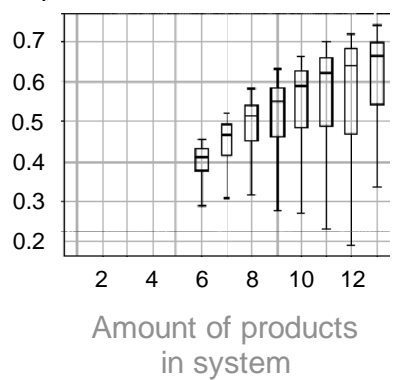

c)

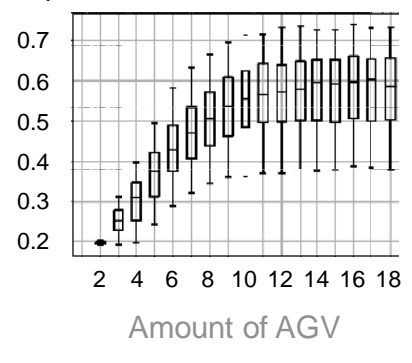

Figure 4: Sensitivity analysis of target parameters in relation to target value. 


\section{Conclusion and Outlook}

Within this paper, a data analysis framework for planning of manufacturing systems was developed and implemented in a use case regarding the material supply strategy within the MMS. The use case shows the consistent implementation of the framework from the running of simulation test series to data analysis of the generated results. With the help of the developed framework, the parameters "Amount of AGV", "Velocity of AGV" and "Amount of products" could be identified as particularly important for the overall utilisation of the manufacturing system. Especially the parameter combinations of 9 to 11 AGV with a velocity between 0.75 and $0.95 \mathrm{~m} / \mathrm{s}$ and 9 to 11 products were identified as optimal to achieve a high overall utilisation of the manufacturing system.

Since the use case only considered five parameters, test series with parameter variations of all subsystems are necessary to overall improve the manufacturing system. Therefore, it is necessary to analyse the combination of the parameters in order to be able to determine this influence on the target value. Moreover, only one material supply strategy for MMS was analysed. In addition, further research is needed regarding the usage of data mining algorithms for planning of flexible manufacturing systems. Using this approach, parameters with significant influence on the target values of the entire system can be identified at an early planning stage. This may, for example, enable greater consideration of interactions with the environment and can be used for the sustainable planning of manufacturing systems.

\section{References}

[1] Koren Y, Shpitalni, M. Design of reconfigurable manufacturing systems. J. Manuf. Syst.,2011. doi: 10.1016/j.jmsy.2011.01.001

[2] Greschke P, Schönemann PM, Thiede S, Herrmann C. Matrix structures for high volumes and flexibility in production systems. Procedia CIRP. 2014; (17): 160-165. doi: 10.1016/j.procir.2014.02.040
[3] Filz MA, Gerberding J, Herrmann C, Thiede S. Analyzing different material supply strategies in matrix-structured manufacturing systems. Procedia CIRP: 2019, (81): 1004-1009. doi: 10.1016/j.procir.2019.03.242

[4] Nyhuis P, Wiendahl H-P, Fiege T, Mühlenbruch H. Materialbereitstellung in der Montage. Montage der Ind. Produktion. 2006; (111): 324-351. doi: 10.1007/3-540-36669-5_10

[5] Seibold Z,Furmans K. Plug \& Play-Fördertechnik in der Industrie 4.0. In: Handbuch Industrie 4.0, Bd.3. Berlin, Heidelberg: Springer; 2017, 3-20.

[6] Banks J, Carson JS, Nelson BL, Nicol D. Discrete-event system simulation. Upper Saddle River, NJ: Prentice Hall; 2010.

[7] Zhou L, Zhang L, Ren L. Modelling and simulation of logistics service selection in cloud manufacturing. Procedia CIRP. 2018; (72): 916-921. doi: 10.1016/J.PROCIR.2018.03.197

[8] Negahban A, Smith JS. Simulation for manufacturing system design and operation: Literature review and analysis. J. Manuf. Syst.. 1014; (33)2. 241-261. doi: 10.1016/j.jmsy.2013.12.007

[9] Schönemann M, Herrmann C, Greschke P, Thiede S. Simulation of matrix-structured manufacturing systems. J. Manuf. Syst.. 2015; (37): 104-112. doi: 10.1016/j.jmsy.2015.09.002

[10] Büth L, Broderius N, Herrmann C, Thiede S. Introducing agent-based simulation of manufacturing system to industrial discrete-event simulation tools. In 2017 IEEE $15^{\text {th }}$ Int. Conf. Ind. Informatics; $2017 \mathrm{Jul}$; Emden, Germany, 3-8: doi: 10.1109/INDIN.2017.8104934

[11] Kern W, Lämmermann H, Bauernhansl T. An integrated logistics concept for a modular assembly system. Procedia Manuf.. 2017; (11): 957-964. doi: 10.1016/j.promfg.2017.07.200.

[12] Fayyad U, Piatetsky-Shapiro G, Smyth P. "Knowledge discovery and data mining: Towards a unifying framework," 1996. https://www.aaai.org/Papers/KDD/ 1996/KDD96-014.pdf.

[13] Cleff T. Deskriptive Statistik und moderne Datenanalyse. Wiesbaden: Springer Gabler; 2012. 